group can be rescued with co-therapy. These data demonstrate that co-therapy is a safe and effective treatment option in the DGH setting.

Competing interests None declared.

\section{PWE-251 DISTRICT GENERAL HOSPITAL EXPERIENCE OF OPTIMISING TREATMENT OUTCOME ON THIOPURINES BY CO-PRESCRIPTION OF ALLOPURINOL IN PATIENTS WITH INFLAMMATORY BOWEL DISEASE}

doi:10.1136/gutjnl-2012-302514d.251

S Cherian,* A Gera, V Saxena, A Loganayagam. Department of Gastroenterology, Queen Elizabeth Hospital NHS Trust, London, UK

Introduction Numerous patients, especially those with elevated thiopurine methyltransferase (TPMT) activity, selectively methylate thiopurine drugs, generating high levels of methylated metabolites and low thioguanine nucleotides. This pattern of metabolism is related to hepatotoxicity and non-response to therapy. Co-prescription of thiopurines (TP) (at 25\% of standard dose) with allopurinol (xanthine oxidase inhibitor) seems to avoid this problem, optimising both metabolite profile and clinical response. British experience on the use of this combination therapy (CT) remains limited. In this study we report a district general hospital (DGH) experience for the indications of toxicity (mainly hepatic) and very high TPMT activity in patients with inflammatory bowel disease (IBD).

Methods Retrospective notes review of patients at a district general hospital treated with CT using 25\% dose of TP and $100 \mathrm{mg}$ allopurinol was undertaken. Particular attention was paid to whether CT overcame the specific problem that prevented thiopurine monotherapy

Results 15 patients (age $24-77$ yrs, male $=6$, Crohn's=6, ulcerative colitis=9) were identified. All 15 patients were on an oral five amino salicylic acid preparation and 12 patients had previously been on a TP. Two patients with fibrotic stricture and one patient with hepatic steatosis were excluded from the analysis. Of those patients receiving co-prescription for side effects (four hepatoxicity and five others: rash, nausea, headache, fatigue), $78 \%$ were able to tolerate CT with complete resolution of liver function abnormality where relevant. Clinical remission was achieved in $100 \%$ of the patients who tolerated CT. In the three patients where CT was commenced for very high TPMT activity, 1 (33\%) developed non-specific side effects (headache, nausea) leading to discontinuation of therapy and $2(67 \%)$ achieved clinical remission.

Conclusion CT with low-dose TP and allopurinol avoids hepatotoxicity and improves chances for clinical remission. CT may also prevent other side effects. CT should be fully utilised in a DGH for hepatoxicity and other side effects. Using CT as first line in those with high TPMT activity remains questionable and requires further scrutiny in a prospective study.

Competing interests None declared.

\section{PWE-252 TRENDS IN IMAGING AND IMPACT OF DIAGNOSTIC MEDICAL RADIATION IN INFLAMMATORY BOWEL DISEASE}

doi:10.1136/gutjnl-2012-302514d.252

${ }^{1} \mathrm{~S}$ Chatu, ${ }^{*} \mathrm{~A}$ Poullis, ${ }^{1} \mathrm{R}$ Holmes, ${ }^{2} \mathrm{R}$ Greenhalgh, ${ }^{1} \mathrm{R}$ Pollok. ${ }^{1}$ Department of Gastroenterology, St George's Hospital, London, UK; ${ }^{2}$ Department of Radiology, St George's Hospital, London, UK

Introduction Increasing use of diagnostic imaging in inflammatory bowel disease has led to concerns about the malignant potential of ionising radiation in a cohort that is already predisposed to malignancy. The aim was to quantify radiation exposure in inflammatory bowel disease patients referred from primary care, determine predictors of high exposure and evaluate temporal changes in imaging at a single centre.

Methods Patients with a diagnosis of Crohn's disease (CD) or ulcerative colitis (UC) were prospectively recruited from clinic between January 2011 and June 2011. Demographic and clinical data were obtained by scrutinising medical records. The number and type of imaging procedures was obtained from the radiology database which was first set up in 1990, we only included those diagnosed after the database was initiated. The effective dose of radiation from each test was estimated from published standardised tables. Cumulative effective dose (CED) was calculated for each subject by summing the effective doses of radiation from diagnosis until end of study period which was June 2011. Cox regression analysis was performed to assess for factors associated with potentially harmful levels of ionising radiation defined as total CED $>50 \mathrm{mSv}$.

Results The cohort included 415 patients. Median disease duration for Crohn's disease and ulcerative colitis was 8.3 and 7.7 years. Median total CED was $7.2 \mathrm{mSv}$ (IOR 3.0-22.7) in Crohn's disease and $2.8 \mathrm{mSv}$ (IOR $0.8-8.9$ ) in ulcerative colitis patients. A total of 32 patients (8\%) received a CED $>50 \mathrm{mSv}$. Multivariate analysis revealed a history of IBD related surgery had a HR of 7.7. During the study period usage of abdominal CT increased by $350 \%$.

Conclusion About 1 in 10 patients were exposed to potentially harmful levels of ionising radiation therefore strategies to reduce radiation exposure are needed. While there was an increased uptake of both MRI and small bowel ultrasound over the past 20 years use of CT also increased substantially.

Competing interests None declared.

\section{PWE-253 ADALIMUMAB IMPROVES HEALTH-RELATED OUALITY OF LIFE FOR 52 WEEKS IN PATIENTS WITH ULCERATIVE COLITIS}

doi:10.1136/gutjnl-2012-302514d.253

${ }^{1} \mathrm{~W} J$ Sandborn, ${ }^{2} \mathrm{G}$ Van Assche, ${ }^{3} \mathrm{R}$ B Thakkar, ${ }^{4} \mathrm{~A}$ Lazar, ${ }^{4} \mathrm{M}$ Kron, ${ }^{3} \mathrm{M}$ Yang ${ }^{5} \mathrm{~S}$ P Patel, ${ }^{*} \mathrm{~J}$ Chao, ${ }^{3} \mathrm{P}$ M Mulani. ${ }^{1}$ UCSD, La Jolla, California, USA; ${ }^{2}$ U Hosp Gasthuisberg, Leuven, Belgium; ${ }^{3}$ Abbott, Abbott Park, Illinois, USA; ${ }^{4}$ Abbott, Ludwigshafen, Germany; ${ }^{5}$ Abbott, Maidenhead, UK

Introduction We investigated effects of adalimumab (ADA) maintenance therapy on health-related quality of life (HRQOL) through 52 weeks (wks) in patients (pts) with ulcerative colitis (UC).

Methods 494 pts with moderate to severe UC (Mayo score, 6-12 points; endoscopic subscore, 2-3 points; anti-tumour necrosis factor [anti-TNF]-naïve and anti-TNF-experienced [40.3\%]) who had failed conventional therapy were enrolled in a $52-w k$, randomised, double-blind, placebo ( $\mathrm{PBO}$ )-controlled maintenance trial. ADA-treated pts received induction therapy (160/80 mg@Wks 0/2) and 40-mg every-other-week (eow) maintenance therapy. Pts with inadequate response could switch to open-label eow therapy after Wk 12 and subsequently to weekly therapy. HROOL was measured by Inflammatory Bowel Disease Questionnaire (IBDQ). Intent-totreat population was analysed. IBDQ response rates were compared between treatment groups using Cochran-Mantel-Haenszel test stratified for prior anti-TNF use whereas $\chi^{2}$ test was used in antiTNF-naïve pts. Non-responder imputation was used for response variables. For change of IBDQ scores, ANCOVA model with treatment and prior anti-TNF status as factors and baseline value as covariate was used. Missing values were imputed through last observation carried forward (LOCF).

Results Significantly more ADA-treated pts were IBDQ responders (increase in IBDQ score $\geq 16$ points from baseline) throughout Wks 